\title{
Polarity correspondence in comparative number magnitude judgments
}

\author{
ROLF REBER \\ University of Bergen, Bergen, Norway \\ Pascal Wurtz \\ Inselspital, Bern, Switzerland \\ AND \\ MARIt KNAPSTAD AND LinN VATHNE LERVIK \\ University of Bergen, Bergen, Norway
}

\begin{abstract}
When asked which of two digits is greater, participants respond more quickly if physical size corresponds to number magnitude, such as in 37 , than when the two attributes contradict each other, such as in 3 7. This size congruence effect in comparative number judgments is a well-documented phenomenon. We extended existing findings by showing that this effect does not depend on physical size of the number alone but can be observed with number symmetry. In addition, we observed that symmetric numbers are judged as being smaller than asymmetric numbers, which renders an interpretation of the number symmetry congruence effect in terms of physical size implausible. We refer to the polarity correspondence principle (Proctor \& Cho, 2006) to explain the present findings.
\end{abstract}

When participants in an experiment are presented with two different digits and have to decide which is greater, they are influenced by differences in the physical size of the digits: They respond more quickly if physical size and numerical magnitude coincide (e.g., 7 3) than when they are incongruent, as in the pair 73 (Besner \& Coltheart, 1979; Henik \& Tzelgov, 1982).

Additional evidence from fMRI data (Pinel, Piazza, Le Bihan, \& Dehaene, 2004) and event-related potentials (Schwarz \& Heinze, 1998) supports the notion that numerical judgments are related to size processing. Moreover, Cohen Kadosh and Henik (2006; Rubinsten \& Henik, 2005) showed that not only physical size, but also figureground contrast affected comparative number judgments: Participants responded more quickly if the brighter of two digits shown on a dark background coincided with the greater numerical value. They explained their findings with the existence of an amodal representation of physical size and numerical magnitude (see Walsh, 2003). Indeed, higher figure-ground contrast may result in the subjective perception of bigger physical size (Weale, 1975).

This size congruence effect in comparative number judgments can be explained in terms of an automatic interference mechanism (Pansky \& Algom, 1999). If the irrelevant attribute draws attention from the relevant attribute, interference occurs. The more salient the irrelevant attribute is, the higher the interference effect on performance of the relevant attribute becomes (Fitousi
\& Algom, 2006; Pansky \& Algom, 1999; see Schwarz $\&$ Ischebeck, 2003, for a similar account). The only irrelevant attribute that has been tested to date is physical size, including luminance, which may result in subjective differences in physical size. Therefore, the question arises as to whether there are other irrelevant attributes that bias comparative number judgments.

In order to provide an answer to this question, we refer to the concept of polarity correspondence to explain response time (RT) differences in speeded binary classification tasks (Proctor \& Cho, 2006): Research has revealed that processing is faster if the polarity of two dimensions is the same. Let us look at the so-called spatial-numerical association of response codes (SNARC) effect as an example. Dehaene, Bossini, and Giraux (1993) found that parity judgments (odd/even) were faster when participants had to respond to relatively small numbers with the left hand and to relatively large numbers with the right hand, which suggests that people represent number magnitude spatially, with lower values represented on the left and higher values on the right. When Ito and Hatta (2004) replicated the study by Dehaene et al. with a vertical arrangement of the response keys, they observed a SNARC effect: Participants answered more quickly to digits of greater numerical magnitude when they had to press the upper key, rather than the lower key. Proctor and Cho explained this finding by invoking their polarity correspondence principle: Both the stimulus attribute greater and 
the response attribute upper (or smaller and lower) have the same polarity. On the basis of an extensive review of experimental studies, the authors concluded that RTs are shorter when stimulus and response attributes have the same polarity than when the attributes have different polarity (greater-lower or smaller-upper).

With linguistic stimuli, polarity correspondence between two stimulus attributes can be derived from linguistic markedness: The unmarked end of a dimension denotes the default form. For example, people are more likely to ask the question "how large is ..." (e.g., the moon) than "how small is ..." (e.g., the moon). Therefore, the term large is the unmarked end for the dimension size, whereas small is marked. The term symmetric is unmarked, whereas the term asymmetric is marked. Proctor and Cho (2006) assign "+ polarity" to the unmarked end and "-polarity" to the marked end. In our examples, there is polarity correspondence between large and symmetric because both have + polarity and between small and asymmetric because both have - polarity. The polarity correspondence account therefore predicts that not only correspondence between physical size and numerical magnitude, but also polarity correspondence between number magnitude and any other stimulus dimension, such as symmetry, will result in faster processing.

In order to test this assumption, we conducted three experiments that demonstrated that symmetry of two-digit numbers resulted in the same congruence effects as those shown for physical size in earlier studies, although symmetric numbers were judged as being smaller than asymmetric numbers. This finding contradicts an interpretation of the congruence effect in terms of an amodal representation of size but supports the broader notion of polarity correspondence between stimulus attributes. The basic question of Experiments 1 and 2 was whether congruence of symmetry of two-digit numbers with numerical magnitude would result in faster responses, supporting the polarity correspondence account. In addition to pairs with one symmetrical and one asymmetrical number, we used pairs of asymmetrical numbers in order to assess SNARC effects superimposed on the effect of polarity correspondence between number magnitude and symmetry.

\section{EXPERIMENT 1}

\section{Method}

Participants. The participants in the experiment were 24 undergraduate students at the University of Bergen (16 of them female; mean age, 22.9 years).

Materials. Pairs of two-digit numbers were presented at the center of the screen. We used 33, 44, 55, 66, and 77 as symmetric numbers; all the symmetrical items had translatory symmetry, which is the repetition of an element without changing its vertical position, $\mathrm{AB} \leftrightarrow \mathrm{AB}$, but not vertical symmetry, which is the mirroring of element along a vertical axis, $\mathrm{AB} \leftrightarrow \mathrm{BA}$ (see Weyl, 1952). There were 35 different pairs of number values, which were also shown in inverse left-right order (e.g., 6466 and 66 64), resulting in 70 number pairs for the experimental trials. Forty pairs differed by one (e.g., 3132 ), and the remaining 30 pairs by two (e.g., 31 33). When the distance was one, there existed, for each pair with a symmetric number, 1 pair with asymmetric numbers only. These pairs were adjacent to the pairs with one symmetric number and resulted in
4 pairs for each decimal unit; for the fifties, for example, the pairs with a distance of one were $5354,5455,5556$, and 5657 . When the distance was two, the pairs adjacent to the pairs with one symmetric number were presented, which included the pair between the 2 pairs with a symmetric number within one decimal unit. For the fifties, these pairs were $5254,5355,5456,5557$, and 5658 . We did not use numbers that ended in 0 , because they may be processed differently (Brysbaert, 1995), or number pairs from different decades, because these may yield interaction effects, as demonstrated in studies on the distance effect in number comparison with two-digit numbers (Ganor-Stern, Tzelgov, \& Ellenbogen, 2007; Verguts \& De Moor, 2005). In order to implement the pairings just discussed, we used pairs from the thirties to the seventies with a distance of one and from the forties to the sixties with a distance of two. Each of the 70 number pairs was shown three times. From 210 experimental trials, there were 114 trials on which both numbers were asymmetric and 96 trials on which one of the numbers was symmetric, half of them on the left side and half on the right side.

Procedure. The participants sat about $55 \mathrm{~cm}$ from a computer screen and had a serial response box (Psychology Software Tools) in front of them. On each trial, a fixation cross (duration, $500 \mathrm{msec}$ ) was shown at the center of the screen, followed by a blank screen (duration, $100 \mathrm{msec}$ ). Then the number pairs were shown until the participants responded. The numbers, written in Courier New, were shown in white on black background and were about $10 \mathrm{~mm}\left(1.0^{\circ}\right)$ high and about $13 \mathrm{~mm}\left(1.4^{\circ}\right)$ wide. The distance between the centers of two numbers was $24 \mathrm{~mm}\left(2.5^{\circ}\right)$. We presented three blocks of 80 trials (10 practice +70 experimental), which resulted in a total of 240 trials. Between the blocks, there was a break of $1 \mathrm{~min}$. The participants had to press the left key of the response box if the left number had the greater value and the right key if the right number had the greater value. They were instructed to respond as quickly as possible, but accurately.

\section{Results and Discussion}

For each participant, we computed the median RT for each of the six symmetry $\times$ greater value conditions. Means and standard deviations for RTs and accuracy across participants are shown in Table 1. This resulted in a $3 \times 2$ factorial ANOVA design, with both symmetry (left, right, or none) and greater value (left or right) manipulated within subjects. The analysis for RTs yielded significant effects of symmetry $[F(2,46)=19.16, p<$ $.001]$ and of greater value $[F(1,23)=12.17, p=.002]$ and an interaction between symmetry and greater value $[F(2,46)=5.26, p=.009]$. Subsequent $t$ tests showed that the participants were faster when the greater of two asymmetric numbers was on the right side than when it was on the left side $[t(23)=4.19, p<.001]$. When the symmetric number was on the right side, the participants were faster when the greater number was on the right side rather than on the left side $[t(23)=2.95, p=.007]$. However, there was no significant difference when the symmetric number was on the left side $[t(23)=0.74]$.

The same ANOVA for accuracy yielded a significant interaction $[F(2,46)=3.98, p=.026]$. Subsequent $t$ tests revealed a pattern similar to that for the RT data: The participants were more accurate when the symmetric number on the right side was greater than when it was smaller $[t(23)=$ $2.60, p<.016]$, but there was no significant difference when the left number was symmetric $[t(23)=1.07]$ or when both numbers were asymmetric $[t(23)=1.82]$.

This experiment showed that congruence between symmetry and number magnitude rendered judgments faster 
Table 1

Means and Standard Deviations for Response Times and Accuracy by Number Symmetry and Side With Greater Numerical Value, Experiment $1(N=24)$

Symmetric Number

\begin{tabular}{|c|c|c|c|c|c|c|}
\hline \multirow{3}{*}{$\begin{array}{c}\text { Greater } \\
\text { Value }\end{array}$} & \\
\hline & \multicolumn{2}{|c|}{ Left } & \multicolumn{2}{|c|}{ Right } & \multicolumn{2}{|c|}{ None } \\
\hline & $M$ & $S D$ & $M$ & $S D$ & $M$ & $S D$ \\
\hline \multicolumn{7}{|c|}{ Response Time } \\
\hline Left & 603 & 98 & 644 & 122 & 661 & 118 \\
\hline Right & 604 & 110 & 603 & 80 & 611 & 86 \\
\hline \multicolumn{7}{|c|}{ Accuracy } \\
\hline Left & .958 & .043 & .933 & .068 & .938 & .058 \\
\hline Right & .937 & .084 & .965 & .057 & .954 & .054 \\
\hline
\end{tabular}

and more accurate, as compared with pairs where the greater number was asymmetric. This finding supports the polarity correspondence account of Proctor and Cho (2006). However, the effect was one-sided in that it appeared only when the symmetric number was on the right side, and the participants reacted more quickly when the right, rather than the left, number in asymmetric pairs was greater. This result might reflect a SNARC effect, because the greater number on the right side elicits faster responses. More important, magnitude and location of the response (left vs. right) were confounded, so that it is not clear whether the observed effect was due to polarity correspondence between symmetry and number magnitude or between symmetry and location of response.

\section{EXPERIMENT 2}

After having shown a congruence effect between number magnitude and symmetry, we repeated the experiment, with the exception that participants were asked to decide which of the numbers was smaller (e.g., Banks, Fujii, \& Kayra-Stuart, 1976; Verguts \& De Moor, 2005). This experiment had two motivations. First, if responses are faster when symmetry coincides with the location of the response, participants should be faster when the smaller of the two numbers is symmetric. In contrast, if responses are faster when symmetry coincides with number magnitude, participants should be slower when the smaller of the two numbers is symmetric. Second, we now could observe whether the difference in asymmetrical number pairs was due to a SNARC effect: If the participants responded more quickly when the smaller of two numbers was on the right side, it could not be a SNARC effect. In contrast, if responses were faster when the smaller of two numbers was on the left side, it probably was due to a SNARC effect, because it reflected the mental number line.

\section{Method}

Participants. Twenty-four undergraduate students from the University of Bergen ( 15 of them female; mean age, 22.08 years) participated in the experiment. Two participants apparently misunderstood the instructions (responded to the larger number) and were excluded from further analyses.

Materials and Procedure. The materials and procedure were identical to those in Experiment 1, with the exception that the participants were instructed to press the key on the side with the smaller number.

\section{Results and Discussion}

We first report the analysis for Experiment 2, before we present a combined analysis for Experiments 1 and 2.

Analysis of Experiment 2. For each participant, we computed the median RT for each of the six symmetry $\times$ smaller value conditions. Means and standard deviations for RTs and accuracy across participants are shown in Table 2; since the participants had to respond to the smaller magnitude, we report the location of the smaller number. This yielded a $3 \times 2$ factorial ANOVA design, with both symmetry (left, right, or none) and smaller value (left or right) manipulated within subjects. The analysis for RTs yielded a significant effect of smaller value $[F(1,21)=$ $5.65, p=.027]$, which was qualified by an interaction between symmetry and smaller value $[F(2,42)=11.47$, $p<.001]$; the effect of symmetry was not significant $[F(2,42)=1.78]$. Subsequent $t$ tests showed that the participants were faster when the smaller of two asymmetric numbers was on the left side than when it was on the right side $[t(21)=2.14, p<.045]$. When the symmetric number was on the right side, the participants were faster when the smaller number was on the left side rather than on the right side $[t(21)=4.45, p<.001]$. There was no difference between the two sides if the symmetric number was on the left side $[t(21)=1.86, p=.077]$.

The same ANOVA for accuracy yielded a significant effect of symmetry $[F(2,42)=4.90, p=.012]$ and a significant interaction $[F(2,42)=7.60, p=.002]$. The effect of smaller value was not significant $[F(1,21)=3.08, p=$ $.065]$. Subsequent $t$ tests revealed that the accuracy data were in line with the RT data: The participants were more accurate when the right number was symmetric but the left number was the smaller, rather than the greater, one $[t(21)=3.87, p<.001]$. There were no significant differences when the left number was symmetric $[t(21)=1.11]$ or when both numbers were asymmetric $[t(21)=0.24]$.

Experiment 2 showed that the participants responded more slowly and less accurately when the symmetric number coincided with the smaller number to which the participants had to respond. In addition, the participants responded more quickly when the left of two asymmetric numbers was smaller; this supports the notion that a SNARC effect was superimposed on the effect of polarity correspondence between symmetry and number magnitude.

Table 2

Means and Standard Deviations for Response Times and Accuracy by Number Symmetry and Side With Smaller Numerical Value, Experiment $2(N=22)$

\begin{tabular}{|c|c|c|c|c|c|c|}
\hline \multirow{3}{*}{$\begin{array}{l}\text { Smaller } \\
\text { Value }\end{array}$} & \multicolumn{6}{|c|}{ Symmetric Number } \\
\hline & \multicolumn{2}{|c|}{ Left } & \multicolumn{2}{|c|}{ Right } & \multicolumn{2}{|c|}{ None } \\
\hline & $M$ & $S D$ & $M$ & $\overline{S D}$ & $M$ & $S D$ \\
\hline \multicolumn{7}{|c|}{ Response Time } \\
\hline Left & 635 & 104 & 600 & 92 & 629 & 99 \\
\hline Right & 607 & 90 & 657 & 92 & 642 & 91 \\
\hline \multicolumn{7}{|c|}{ Accuracy } \\
\hline Left & .961 & .054 & .978 & .034 & .944 & .051 \\
\hline Right & .976 & .032 & .924 & .069 & .946 & .048 \\
\hline
\end{tabular}


Table 3

Means and Standard Deviations for Response Times and Accuracy by Number Symmetry and Side With Greater Numerical Value, Combined for Experiments 1 and $2(N=46)$

\begin{tabular}{|c|c|c|c|c|c|c|}
\hline \multirow{3}{*}{$\begin{array}{c}\text { Greater } \\
\text { Value }\end{array}$} & \multicolumn{6}{|c|}{ Symmetric Number } \\
\hline & \multicolumn{2}{|c|}{ Left } & \multicolumn{2}{|c|}{ Right } & \multicolumn{2}{|c|}{ None } \\
\hline & $M$ & $S D$ & $M$ & $S D$ & $M$ & $S D$ \\
\hline \multicolumn{7}{|c|}{ Response Time } \\
\hline Left & 605 & 93 & 651 & 108 & 652 & 105 \\
\hline Right & 619 & 107 & 602 & 88 & 620 & 92 \\
\hline \multicolumn{7}{|c|}{ Accuracy } \\
\hline Left & .967 & .039 & .929 & .068 & .938 & .058 \\
\hline Right & .948 & .072 & .971 & .048 & .949 & .053 \\
\hline
\end{tabular}

Combined analysis. In order to further analyze polarity correspondence between symmetry and number magnitude, we combined the data of the two experiments. Means and standard deviations for RTs and accuracy are shown in Table 3. Note that in Experiment 2, the participants had to respond to the smaller number. Since we were interested in polarity correspondence between symmetry and number magnitude, for the combined analysis, we recoded the data of Experiment 2 so that they fitted the format of Experiment 1: For example, the average in Table 3 for the cell where the right number is both symmetric and greater $(602 \mathrm{msec})$ is from the same cell in Table $1(603 \mathrm{msec})$ and from the cell in Table 2 where the right number is symmetric and the left number smaller (600 $\mathrm{msec})$; in a number pair stimulus, the right number must be greater when the left number is smaller.

This yielded a $2 \times 3 \times 2$ factorial ANOVA design, with kind of judgment (greater vs. smaller) manipulated between subjects and symmetry (left, right, or none) and greater value (left or right) manipulated within subjects. The analysis for RTs yielded significant effects for greater value $[F(1,44)=17.24, p<.001]$ and symmetry $[F(2,88)=13.28, p<.001]$ and a significant symmetry $\times$ greater value interaction $[F(2,88)=14.56, p<$ $.001]$. Other effects were not significant $(F \mathrm{~s}>2.60, p \mathrm{~s}>$ .08 ). Subsequent $t$ tests showed that the participants were faster when the greater of two asymmetric numbers was on the right side than when it was on the left side $[t(45)=$ $4.42, p<.001]$. When the symmetric number was on the right side, the participants were faster when the greater number was on the right side rather than on the left side $[t(45)=5.14, p<.001]$. There was no difference between the two sides if the symmetric number was on the left side $[t(45)=1.39]$.

The same ANOVA for accuracy yielded a significant symmetry $\times$ greater value interaction $[F(2,88)=10.38$, $p<.001]$. Other effects were not significant $(F \mathrm{~s}>3.42$, $p \mathrm{~s}>$.07). Subsequent $t$ tests revealed that the accuracy data were in line with the RT data: The participants were more accurate when the symmetric number on the right was greater than when it was smaller $[t(45)=4.57, p<$ $.001]$, but not when the left number was symmetric or when both numbers were asymmetric $[t(45)<1.52]$.

As the combined analysis makes clear, the participants were faster when the symmetric number was greater, irre- spective of the experimental instruction to respond to the greater number in Experiment 1 or to the smaller number in Experiment 2. This experiment supports the notion of polarity correspondence between symmetry and number magnitude. A SNARC effect was superimposed on the symmetry-magnitude congruence effect. As was noted in the introduction, this SNARC effect can be explained by polarity correspondence between number magnitude and side of response (see Proctor \& Cho, 2006).

An alternative possibility is that symmetric numbers are seen as being physically larger, which would mean that our findings fit an amodal size account (Cohen Kadosh \& Henik, 2006). Therefore, by assessing magnitude judgments for the two-digit numbers used in Experiments 1 and 2, we examined the possibility that symmetric numbers are seen as being larger than asymmetric numbers.

\section{EXPERIMENT 3}

\section{Method}

Participants. Twenty-seven undergraduate students from the University of Bergen (20 of them female; mean age, 20.07 years) participated in the experiment.

Materials and Procedure. A single number was shown in the center of the screen. Each number was shown in four different sizes: $15,16,17$, or 18 points. This resulted in 20 ( 4 repetitions $\times 5$ ) symmetric numbers and 104 (4 repetitions $\times 26$ ) asymmetric numbers, for a total of 124 trials. As in Reber, Zimmermann, and Wurtz (2004), the participants were instructed to rate the font size of the stimuli on a scale from 1 (small) to 9 (large). In order to give an idea how large the stimuli were, we presented 12 practice trials with numbers used in the practice trials of Experiments 1 and 2 before the experimental trials started.

\section{Results and Discussion}

For each participant, we computed the mean rating for each of the symmetry $\times$ font size conditions, as plotted in Figure 1. A $2 \times 4$ factorial ANOVA, with both symmetry (symmetric vs. asymmetric) and font size $(15,16$, 17 , or 18 points) manipulated within subjects, yielded a main effect of font size $[F(3,78)=50.60, p<.001]$. More important, the participants perceived symmetric numbers as being smaller than asymmetric numbers $[F(1,26)=$

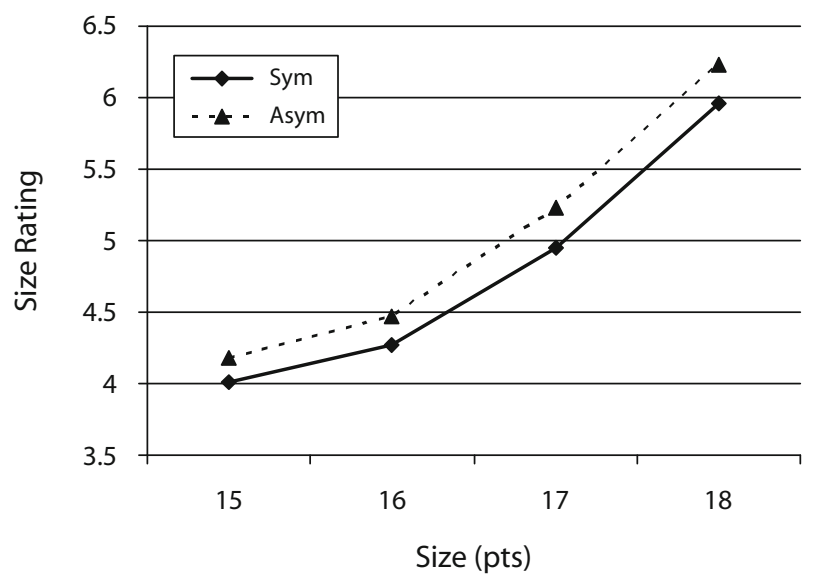

Figure 1. Size estimates for symmetric and asymmetric numbers for the assessment of the materials in Experiment 3. 
$15.78, p=.001]$. The symmetry $\times$ font size interaction was not significant $[F(3,78)=0.24]$.

In sum, symmetric numbers were judged as being smaller than asymmetric numbers. Therefore, the observed congruence effects between symmetry and number magnitude cannot be explained in terms of physical size and, therefore, support the broader polarity correspondence account.

\section{GENERAL DISCUSSION}

We manipulated the symmetry of two-digit numbers and instructed the participants to respond on the side of the greater numerical value (Experiment 1). Responses were faster if the greater number was symmetric. This was also the case when we instructed the participants to press the key on the side of the smaller number (Experiment 2). This finding cannot be explained by the proposed mechanism that links physical size to number magnitude (Cohen Kadosh \& Henik, 2006; Dehaene, 1997): In fact, symmetric two-digit numbers are seen as being smaller than asymmetric two-digit numbers (Experiment 3).

These findings extend existing research on the size congruence effect by showing that any salient stimuluswhich means that they possess + polarity-is associated with greater numerical magnitude. Whereas a higher figure-ground contrast of the stimulus may result in subjectively larger stimulus size, this was definitely not the case with symmetry. Symmetric numbers were seen as being smaller than asymmetric numbers, an effect that has been replicated under different conditions (Reber, Meier, Christensen, Lervik, Teige, \& Mitterndorfer, unpublished data).

Fitousi and Algom (2006) found that congruence between the size of lines separated from the number stimuli and number magnitude resulted in shorter RTs if the irrelevant dimension was more salient. Our findings support this notion with stimulus material that is unrelated to size. Earlier studies and theoretical accounts dealt with the size congruence effect. The important insight of the present study lies in the demonstration that attributes unrelated to physical size can interfere with judgment of number magnitude; therefore, physical size is not a necessary ingredient in an account of this effect. The broader principle of polarity correspondence - introduced by Proctor and Cho (2006) - fits well with the observed pattern of results.

\section{AUTHOR NOTE}

This research was funded by the Norwegian Research Council, Grant 192415, to R.R. We thank Kenneth Hugdahl and Bjørn Sætrevik for comments on earlier versions of the manuscript. Correspondence concerning this article should be addressed to R. Reber, Department of Biological and Medical Psychology, University of Bergen, Postboks 7800, N-5020 Bergen, Norway (e-mail: rolf.reber@psysp.uib.no).

\section{REFERENCES}

Banks, W. P., Fujit, M., \& Kayra-Stuart, F. (1976). Semantic congruity effects in comparative judgments of magnitudes of digits. Journal of Experimental Psychology: Human Perception \& Performance, 2, 435-447. doi:10.1037/0096-1523.2.3.435

Besner, D., \& Coltheart, M. (1979). Ideographic and alphabetic processing in skilled reading of English. Neuropsychologia, 17, 467-472. doi:10.1016/0028-3932(79)90053-8

BRYSBAERT, M. (1995). Arabic number reading: On the nature of the numerical scale and the origin of phonological recoding. Journal of Experimental Psychology: General, 124, 434-452. doi:10.1037/0096 $-3445.124 .4 .434$

Cohen Kadosh, R., \& Henik, A. (2006). A common representation for semantic and physical properties: A cognitive-anatomical approach. Experimental Psychology, 53, 87-94. doi:10.1027/1618 $-3169.53 .2 .87$

DeHAene, S. (1997). The number sense: How the mind creates mathematics. Oxford: Oxford University Press.

Dehaene, S., Bossini, S., \& Giraux, P. (1993). The mental representation of parity and number magnitude. Journal of Experimental Psychology: General, 122, 371-396. doi:10.1037/0096-3445.122.3.371

Fitousi, D., \& Algom, D. (2006). Size congruity effects with two-digit numbers: Expanding the number line? Memory \& Cognition, 34, 445 457.

Ganor-Stern, D., Tzelgov, J., \& Ellenbogen, R. (2007). Automaticity and two-digit numbers. Journal of Experimental Psychology: Human Perception \& Performance, 33, 483-496. doi:10.1037/0096 $-1523.33 .2 .483$

HeniK, A., \& Tzelgov, J. (1982). Is three greater than five: The relation between physical and semantic size in comparison tasks. Memory \& Cognition, 10, 389-395.

Iто, Y., \& HatтA, T. (2004). Spatial structure of quantitative representation of numbers: Evidence from the SNARC effect. Memory \& Cognition, 32, 662-673.

PANSKY, A., \& AlgOM, D. (1999). Stroop and Garner effects in comparative judgment of numerals: The role of attention. Journal of Experimental Psychology: Human Perception \& Performance, 25, 39-58. doi:10.1037/0096-1523.25.1.39

Pinel, P., Piazza, M., Le Bihan, D., \& Dehaene, S. (2004). Distributed and overlapping cerebral representations of number, size, and luminance during comparative judgments. Neuron, 41, 983-993. doi:10.1016/S0896-6273(04)00107-2

Proctor, R. W., \& Cho, Y. S. (2006). Polarity correspondence: A general principle for performance of speeded binary classification tasks. Psychological Bulletin, 132, 416-442. doi:10.1037/0033 $-2909.132 .3 .416$

Reber, R., Zimmermann, T. D., \& Wurtz, P. (2004). Judgments of duration, figure-ground contrast, and size for words and nonwords. Perception \& Psychophysics, 66, 1105-1114.

Rubinsten, O., \& HeniK, A. (2005). Automatic activation of internal magnitudes: A study of developmental dyscalculia. Neuropsychology, 19, 641-648. doi:10.1037/0894-4105.19.5.641

Schwarz, W., \& Heinze, H.-J. (1998). On the interaction of numerical and size information in digit comparison: A behavioral and event-related potential study. Neuropsychologia, 36, 1167-1179. doi:10.1016/S0028-3932(98)00001-3

ScHWARZ, W., \& IscheBEcK, A. (2003). On the relative speed account of number-size interference in comparative judgments of numerals. Journal of Experimental Psychology: Human Perception \& Performance, 29, 507-522. doi:10.1037/0096-1523.29.3.507

Verguts, T., \& De Moor, W. (2005). Two-digit comparison: Decomposed, holistic, or hybrid? Experimental Psychology, 52, 195-200. doi:10.1027/1618-3169.52.3.195

WALSH, V. (2003). A theory of magnitude: Common cortical metrics of time, space and quantity. Trends in Cognitive Sciences, 7, 483-488. doi:10.1016/j.tics.2003.09.002

Weale, R. A. (1975). Apparent size and contrast. Vision Research, 15 949-955. doi:10.1016/0042-6989(75)90235-7

Weyl, H. (1952). Symmetry. Princeton, NJ: Princeton University Press.

(Manuscript received May 23, 2009; revision accepted for publication November 1, 2009.) 